\title{
PHARMACOKINETICS LIMITS CLINICAL USE OF IVERMECTIN FOR COVID-19
}

\author{
Ricardo Pena Silva ${ }^{1}$ and Sandra Jaramillo Rincon ${ }^{1}$ \\ ${ }^{1}$ Universidad de los Andes
}

April 28, 2020

\begin{abstract}
NA

Hundreds of researchers are working on developing a vaccine or testing drugs to mitigate COVID-19 worldwide. If novel compounds are found, geopolitical and economic variables will determine their introduction to communities. Therefore, finding low-cost and accessible substances for prevention or treatment of COVID-19 would be ideal.
\end{abstract}

Earlier in April, a new hope emerged when the antiviral effect of ivermectin, a known anti-parasite drug, upon SARS-CoV-2 was published. Caly et al unveiled that $5 \mathrm{uM}$ ivermectin induces a profound reduction ( 5000 fold) in SARS-CoV-2 replication (RNA levels) in cultured human cells ${ }^{1}$. The authors suggested that this drug could reduce viral load in infected patients, with a potential effect on disease progression and spread. Amidst fear of the pandemic, the public and some physicians may be tempted to use ivermectin as prophylaxis, or as a coadjuvant, for COVID-19. These actions have motivated cautionary statements from institutions such as the FDA against the use of pharmaceutical formulations of ivermectin, intended for animals, as therapeutics in humans ${ }^{2}$.

It is vital to be careful with the translation of molecular findings into clinical outcomes, and it is especially important to understand the pharmacokinetic profile of drugs that could be repurposed for COVID-19, in order to design optimal dosing regimens ${ }^{3}$. There is no evidence that the concentration of ivermectin used in this study can be achieved in humans. Multiple teams have evaluated the pharmacokinetics of ivermectin in humans ${ }^{4-6}$ (fig 1), and protocols using the highest doses (approx. $1800 \mu \mathrm{g} / \mathrm{kg}$ and about 10 times the usual dose), have achieved maximum plasma concentrations of about $0.28 \mu \mathrm{M}^{6}$. Therefore, the highest concentration reached is 17.5 times lower than what is required to reduce the replication of SARS-CoV-2. Consequently, although ivermectin may have anin vitro antiviral effect, it probably will not effectivein vivo . Pharmacokinetics may explain lack of effectiveness of ivermectin $(400 \mu \mathrm{g} / \mathrm{kg}$ for 3 days $)$ for treatment of viral infections such as dengue fever ${ }^{7}$.

These results should not discourage us. We do not know what the ceiling concentration of ivermectin in humans is, and administering higher doses of ivermectin may be useful, but could also increase the risk of adverse effects. Besides, some more potent ivermectin analogs may also have an antiviral effect on SARS$\mathrm{CoV}-2$, although this idea requires further study. In summary, it is crucial to be cautious and consider the clinical pharmacokinetics of potential treatments for COVID-19 before initiating off-label therapies in communities and health care workers.

\section{REFERENCES}


1. Caly L, Druce JD, Catton MG, Jans DA, Wagstaff KM. The FDA-approved Drug Ivermectin inhibits the replication of SARS-CoV-2 in vitro.Antiviral Res. 2020:104787.

2. Solomon S. FDA Letter to Stakeholders: Do Not Use Ivermectin Intended for Animals as Treatment for COVID-19 in Humans. 2020; https://www.fda.gov/animal-veterinary/product-safety-information/fda-letterstakeholders-do-not-use-ivermectin-intended-animals-treatment-covid-19-humans. Accessed April 15th, 2020.

3. Smith PF, Dodds M, Bentley D, Yeo K, Rayner C. Dosing will be a key success factor in repurposing antivirals for COVID-19. Br J Clin Pharmacol. 2020.

4. Smit MR, Ochomo EO, Waterhouse D, et al. Pharmacokinetics-Pharmacodynamics of High-Dose Ivermectin with Dihydroartemisinin-Piperaquine on Mosquitocidal Activity and QT-Prolongation (IVERMAL). Clin Pharmacol Ther.2019;105(2):388-401.

5. Duthaler U, Suenderhauf C, Karlsson MO, et al. Population pharmacokinetics of oral ivermectin in venous plasma and dried blood spots in healthy volunteers. Br J Clin Pharmacol.2019;85(3):626-633.

6. Guzzo CA, Furtek CI, Porras AG, et al. Safety, tolerability, and pharmacokinetics of escalating high doses of ivermectin in healthy adult subjects. J Clin Pharmacol. 2002;42(10):1122-1133.

7. Yamasmith E, Saleh-arong FA, Avirutnan P, et al. Efficacy and Safety of Ivermectin against Dengue Infection: A Phase III, Randomized, Double-blind, Placebo-controlled Trial. The 34th Annual Meeting The Royal College of Physicians of Thailand; April, 2018, 2018; PEACH Royal Cliff Beach Resort, Pattaya, Chonburi, Thailand

\section{FIGURE LEGEND:}

Figure 1: Maximal concentrations of ivermectin in plasma of treated subjects. Data pooled from refs ${ }^{4-6}$. When necessary, an estimated body weight of $65 \mathrm{~kg}$ was used for calculations. Note that none of the doses reached the $5 \mu \mathrm{M}$ concentration required for the antiviral effect of ivermectin.

\section{Ivermectin $\mathrm{C}_{\max }$ in Clinical Trials}

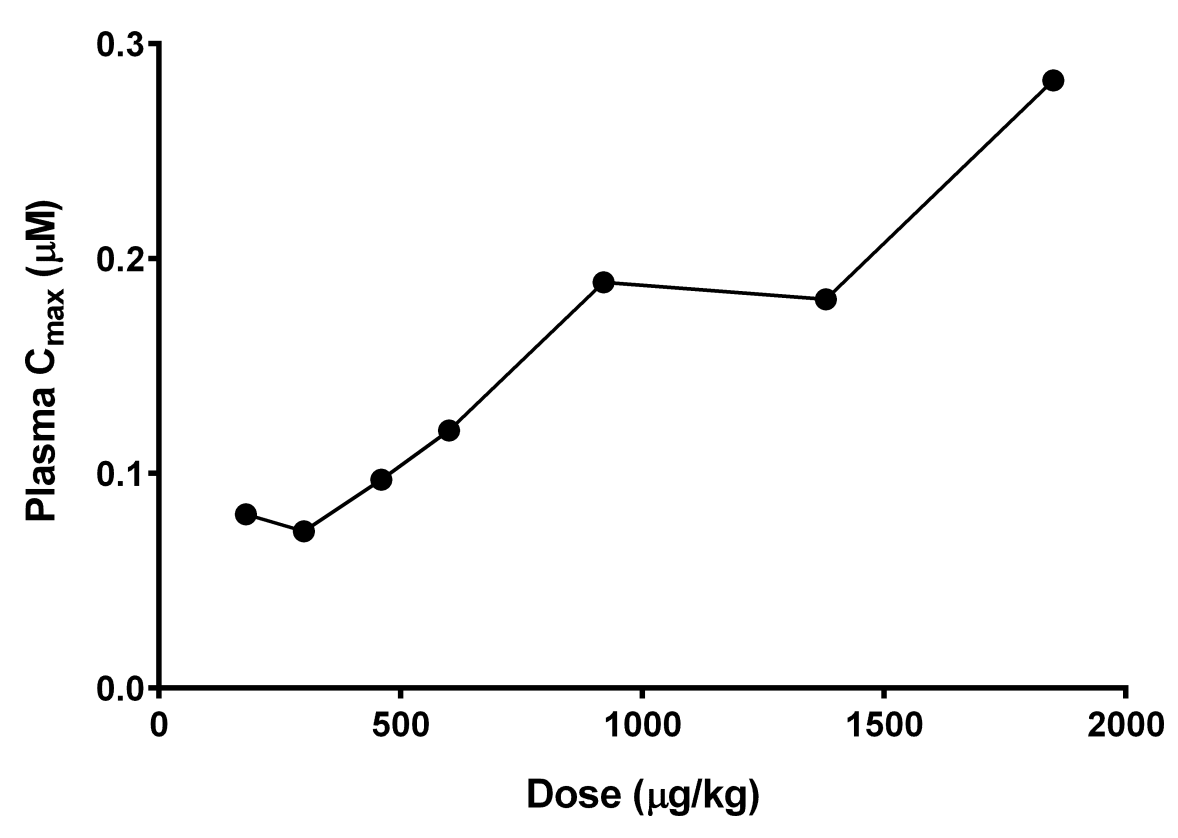

